\title{
Unterscheidung und Quantifizierung von Geruchsstoffen im ppb-Bereich mit temperaturzyklisch betriebenen MOS- Sensoren
}

\author{
Julian Joppich, Andreas Schütze, Tilman Sauerwald \\ Lehrstuhl für Messtechnik, Universität des Saarlandes, 66123 Saarbrücken \\ j.joppich@Imt.uni-saarland.de
}

\begin{abstract}
Zusammenfassung
Eine Methode zur Messung von zwei geruchsintensiven Gasen in Luft, Schwefelwasserstoff und Dimethylsulfid, wird präsentiert. Die verwendeten Verdünnungen im ppb-Bereich entsprechen Geruchsstoffkonzentrationen von ca. 20-250 GE/m3. Die Messung erfolgt mit einem temperaturzyklisch betriebenen Halbleiter-Gassensor. Mithilfe des DSR-Modells (differential surface reduction) werden Merkmale des transienten Leitwertsignals extrahiert. Die Merkmale werden bezüglich ihrer Temperaturabhängigkeit und der Möglichkeit, die Geruchsstoffkonzentration zu quantifizieren, untersucht. Über die unterschiedliche Temperaturabhängigkeit der Merkmale können die Zielgase voneinander unterschieden werden. Neben einer stoffspezifischen oder gemeinsamen Quantifizierung unter Zuhilfenahme aller Merkmale ist unter den gegebenen Bedingungen auch eine gemeinsame Quantifizierung für beide Gase bei ausschließlicher Nutzung des Merkmals einer bestimmten Temperaturstufe des Temperaturzyklus möglich.
\end{abstract}

Keywords: Gassensor, Temperaturzyklus, Schwefelverbindungen, Quantifizierung, Geruch

\section{Einleitung}

Gerüche haben einen großen Einfluss auf die (empfundene) Luftqualität und das Wohlbefinden der Menschen. Im Gegensatz zu den meisten anderen Messgrößen werden Geruchsstoffkonzentration und Geruchsintensität immer noch ausschließlich auf eine humansensorische Messung, also eine Bewertung durch menschliche Prüfer, zurückgeführt [1]. In vielen Anwendungsfeldern (z. B. der Bewertung von geruchsintensiven Anlagen [2, 3]) wäre eine kontinuierliche Bewertung der Luftqualität mit einem Sensorsystem als Ergänzung dieser gesetzlichen Methode vorteilhaft und würde den Anwendungsbereich von Geruchsmessungen erheblich erweitern. Hierzu müssen die relevanten Geruchsstoffe in sehr geringen Konzentrationen selektiv vor variablem geruchsirrelevantem Hintergrund erkannt und die modellbasierte Vorhersage aus Messdaten mit humansensorischen Bewertungen zur An/Abwesenheit von Geruch und zur Geruchsintensität korreliert werden. Ein wichtiger Anwendungsfall sind Anlagen zur Abwasserreinigung, in denen primär Schwefelverbindungen zur Geruchsemission beitragen [4]. Zur Bewertung müssen geringe Konzentrationen im ppb-Bereich innerhalb von
$10 \mathrm{~s}$ gemessen werden, weshalb MetalloxidHalbleiter-Gassensoren (MOS) mit temperaturzyklischem Betrieb („virtuelles Sensorarray“) verwendet werden, die eine sehr hohe Empfindlichkeit und Selektivität aufweisen [5].

\section{Experimentelle Methoden}

Die Gasangebote werden mit einer Gasmischanlage erzeugt. Als Basis wird katalytisch gereinigte trockene Nulluft verwendet, der eine definierte Feuchte $(50 \%)$ sowie ein realistischer Hintergrund an Wasserstoff (500 ppb) und Kohlenmonoxid (150 ppb) hinzugefügt werden („Normalluft"). Der Normalluft werden die Probengase Schwefelwasserstoff $\left(\mathrm{H}_{2} \mathrm{~S}\right)$ und Dimethylsulfid (DMS) mittels eines zweistufigen Mischers [6] hinzudosiert, sodass sich definierte Konzentrationen zwischen 8,2 und 102,5 ppb $\left(\mathrm{H}_{2} \mathrm{~S}\right)$ beziehungsweise zwischen 7,6 und 95,0 ppb (DMS) ergeben. Es wird ein kommerziell erhältlicher MOS-Gassensor (ASMLV-P2, ams Sensor Solutions Germany) verwendet, der mit einer speziellen Elektronik [7] temperaturzyklisch betrieben wird. Der Temperaturzyklus ist für die Auswertung nach der DSR Methode optimiert [8]. Dabei wird der Sensor mehrfach in Phasen hoher Temperatur betrieben, in denen die Sensoroberfläche oxidiert wird. Nach einer 
(a)

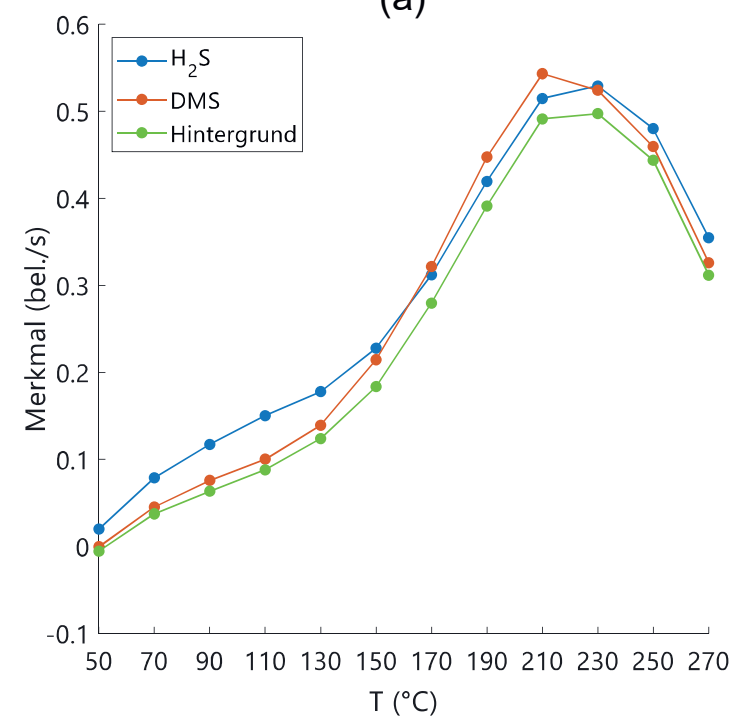

(b)

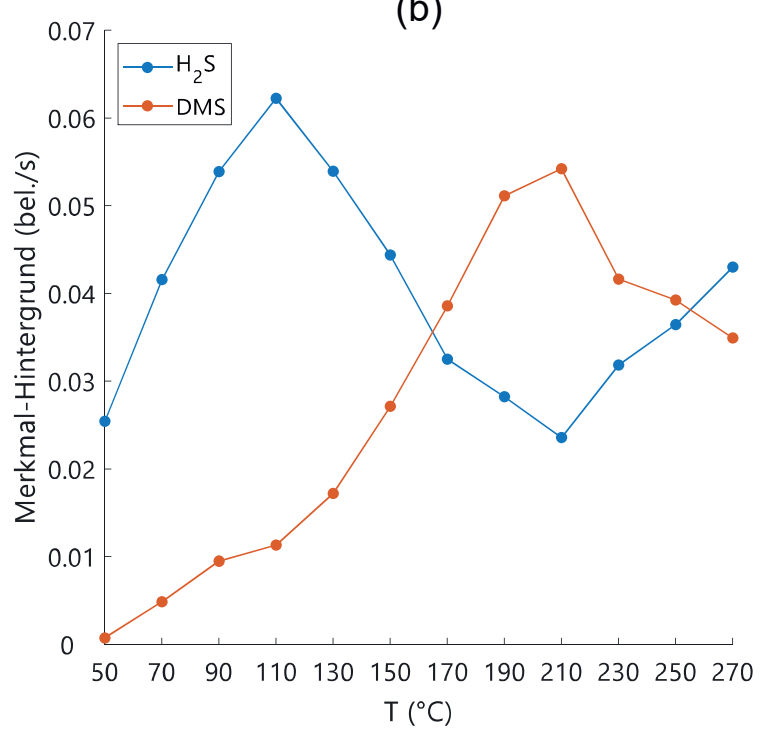

Abb. 1: Temperaturabhängigkeit des Merkmals (Steigung der verschiedenen Temperaturstufen) für $\mathrm{H}_{2} \mathrm{~S}$ und DMS ohne Abzug des Hintergrunds (verursacht v. a. durch $\mathrm{H}_{2}$ und $\mathrm{CO}$ ) (a) und nach Abzug des Hintergrunds (b) bei $100 \mathrm{GE} / \mathrm{m}^{3}$. Durch Abzug des Hintergrunds wird der unterschiedliche Temperaturverlauf der beiden Gase deutlich. Es handelt sich jeweils um Mittelwerte aus mehreren Temperaturzyklen.

schnellen Abkühlung auf eine geringere Temperatur bildet sich die Oxidation der Oberfläche nur langsam zurück. In dieser (kurzen) Phase nach einer Abkühlung ist die Reaktion von reduzierenden Gasen mit dem Oberflächensauerstoff bevorzugt, wodurch der Sensorleitwert steigt [5]. Auf jede der zwölf Hochtemperaturstufen von $450^{\circ} \mathrm{C}$ folgt eine der zwölf äquidistanten Niedertemperaturstufen mit Temperaturen von $50{ }^{\circ} \mathrm{C}$ bis $270^{\circ} \mathrm{C}$ (vgl. Tabelle 1). Die Dauer der Hochtemperaturstufe beträgt $1 \mathrm{~s}$ bis $3 \mathrm{~s}$ und ist so bemessen, dass der Sensor einen stabilen Gleichgewichtswert erreichen kann. Die Dauer der Niedertem-

Tab. 1: Übersicht über die Temperaturstufen und die Zeiten der extrahierten Merkmale

\begin{tabular}{|r|r|r|r|}
\hline$t\left(450{ }^{\circ} \mathrm{C}\right)$ & $T$ & $t(T)$ & $t_{\text {fit }}$ (ab Sprung $)$ \\
\hline $1 \mathrm{~s}$ & $50^{\circ} \mathrm{C}$ & $30 \mathrm{~s}$ & $0,91-1,64 \mathrm{~s}$ \\
\hline $1 \mathrm{~s}$ & $70^{\circ} \mathrm{C}$ & $30 \mathrm{~s}$ & $0,46-0,94 \mathrm{~s}$ \\
\hline $1 \mathrm{~s}$ & $90^{\circ} \mathrm{C}$ & $25 \mathrm{~s}$ & $0,50-0,83 \mathrm{~s}$ \\
\hline $1 \mathrm{~s}$ & $110^{\circ} \mathrm{C}$ & $25 \mathrm{~s}$ & $0,47-1,01 \mathrm{~s}$ \\
\hline $2 \mathrm{~s}$ & $130^{\circ} \mathrm{C}$ & $20 \mathrm{~s}$ & $0,50-1,46 \mathrm{~s}$ \\
\hline $2 \mathrm{~s}$ & $150^{\circ} \mathrm{C}$ & $20 \mathrm{~s}$ & $0,51-3,22 \mathrm{~s}$ \\
\hline $2 \mathrm{~s}$ & $170^{\circ} \mathrm{C}$ & $15 \mathrm{~s}$ & $0,53-2,43 \mathrm{~s}$ \\
\hline $2 \mathrm{~s}$ & $190^{\circ} \mathrm{C}$ & $15 \mathrm{~s}$ & $0,55-1,28 \mathrm{~s}$ \\
\hline $3 \mathrm{~s}$ & $210^{\circ} \mathrm{C}$ & $10 \mathrm{~s}$ & $0,54-0,87 \mathrm{~s}$ \\
\hline $3 \mathrm{~s}$ & $230^{\circ} \mathrm{C}$ & $10 \mathrm{~s}$ & $0,46-0,89 \mathrm{~s}$ \\
\hline $3 \mathrm{~s}$ & $250^{\circ} \mathrm{C}$ & $5 \mathrm{~s}$ & $0,36-0,71 \mathrm{~s}$ \\
\hline $3 \mathrm{~s}$ & $270^{\circ} \mathrm{C}$ & $5 \mathrm{~s}$ & $0,37-0,75 \mathrm{~s}$ \\
\hline
\end{tabular}

peraturstufen beträgt je nach Temperatur $5 \mathrm{~s}$ bis $30 \mathrm{~s}$. Der Sensorleitwert wird bei konstanter Spannung über eine Strommessung mit einem Logarithmier-Verstärker [7] mit einer Abtastrate von $1 \mathrm{kHz}$ gemessen

\section{Ergebnisse und Diskussion}

Mit Hilfe der Datenauswertungs-Toolbox $\mathrm{DAV}^{3} \mathrm{E}$ [9] werden aus den Messdaten die Geruchsstoffe identifiziert und inre Konzentrationen bestimmt. Für die Vorverarbeitung wird das DSR-Modell [7] verwendet, bei dem die Steigung des logarithmierten Leitwerts kurz nach dem Temperatursprung berechnet und als Merkmal extrahiert wird (vgl. Tabelle 1, $t_{\text {fit }}$ beschreibt den Zeitbereich nach dem Temperatursprung, für den die Steigung berechnet wird). In dieser Phase dominiert die Reaktion des Sauerstoffs mit reduzierenden Gasen, die Signalsteigung ist in erster Näherung proportional zur Ratenkonstante [5]. Im Folgenden werden die Konzentrationen in Relation zur Geruchsschwelle des jeweiligen Gases gesetzt, um eine Einordnung der entsprechenden zu bewertenden Geruchsstoffkonzentration zu erhalten. Geruchsstoffkonzentrationen können eigentlich nur durch olfaktorische Messungen bestimmt werden, für die hier verwendeten riechenden Gasmischungen aus jeweils einem verdünnten Reinstoff wird die Geruchsstoffkonzentration jedoch abgeschätzt als Quotient der Volumenkonzentration und der Geruchsschwelle des jeweiligen geruchsintensiven 
(a)

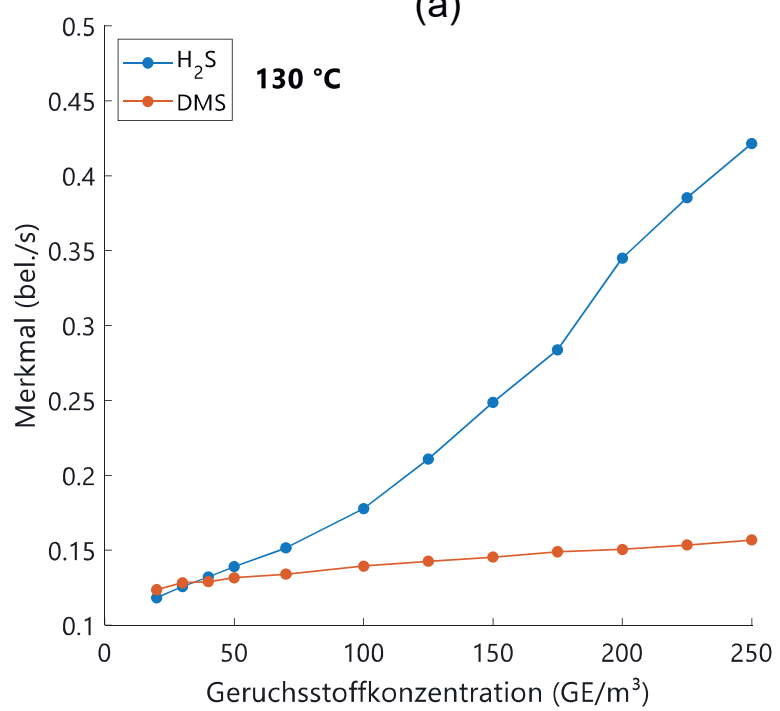

(b)

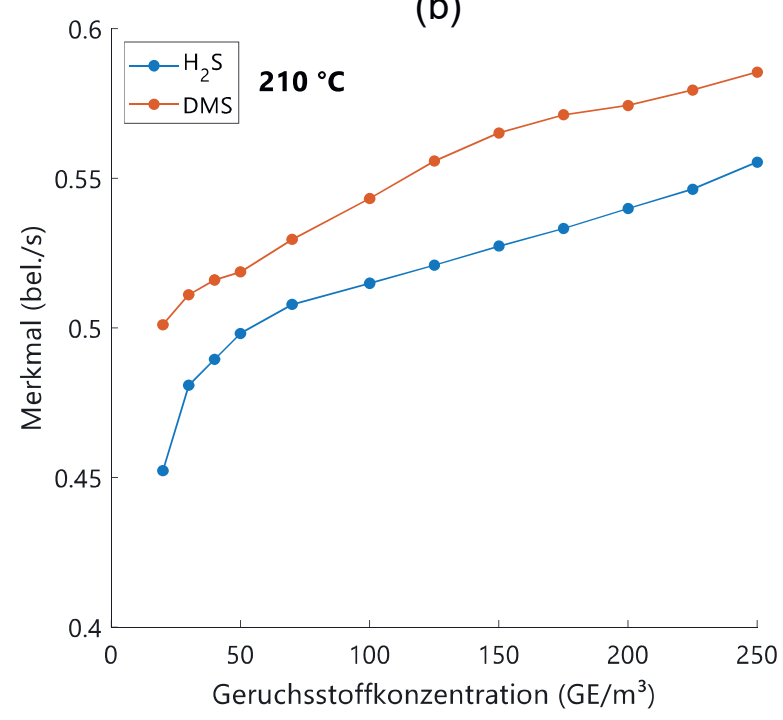

Abb. 2: Beispiele der Konzentrationsabhängigkeit der DSR-Steigung beider Gase bei 130 und $210^{\circ} \mathrm{C}$ (a bzw. b). Auch die meisten anderen Temperaturen zeigen für beide Gase einen deutlich unterschiedlichen Verlauf des Merkmals über die Konzentration. Nur für die Temperaturstufe bei $230{ }^{\circ} \mathrm{C}$ ist die Empfindlichkeit für beide Gase ähnlich (vgl. Abbildung 3). Auch hier wurden Mittelwerte aus mehreren Temperaturzyklen berechnet.

Gases. Sie wird in $\mathrm{GE} / \mathrm{m}^{3}$ angegeben (GE: Geruchseinheiten). Die Geruchsschwelle (per Definition $1 \mathrm{GE} / \mathrm{m}^{3}$ ) liegt für $\mathrm{H}_{2} \mathrm{~S}$ bei einer Konzentration von 0,41 ppb [10] und für DMS bei einer Konzentration von 0,38 ppb [11].

Das Temperaturverhalten der über mehrere Durchläufe des Temperaturzyklus gemittelten Merkmale von $\mathrm{H}_{2} \mathrm{~S}$ und DMS ist in Abbildung 1 a für eine Geruchsstoffkonzentration von $100 \mathrm{GE} / \mathrm{m}^{3}$ gezeigt. Der Untergrund ist durch die ebenfalls reduzierenden Hintergrundgase $\mathrm{H}_{2}$ und $\mathrm{CO}$ dominiert. Dieser Anteil ist in Abbildung $1 \mathrm{~b}$ abgezogen. Es zeigt sich ein deutlich unterschiedlicher Verlauf für beide Gase. Während $\mathrm{H}_{2} \mathrm{~S}$ ein Maximum bei $110^{\circ} \mathrm{C}$ besitzt, liegt dieses bei DMS erst bei einer Temperatur von circa $210^{\circ} \mathrm{C}$.

Die einzelnen Merkmale zeigen über die Geruchsstoffkonzentration ein in guter Näherung lineares Verhalten und eignen sich damit gut zur Quantifizierung von Einzelkomponenten. Bei den meisten Temperaturen sind deutliche Unterschiede zwischen den Zielgasen festzustellen, wie in Abbildung 2 beispielhaft für 130 und $210^{\circ} \mathrm{C}$ dargestellt. Für das DSR-Merkmal bei $230^{\circ} \mathrm{C}$ zeigt sich hingegen eine ähnliche Empfindlichkeit für $\mathrm{H}_{2} \mathrm{~S}$ und DMS im Bereich von 40 bis $250 \mathrm{GE} / \mathrm{m}^{3}$ (Abbildung 3), nur für die beiden kleinsten Geruchsstoffkonzentrationen (20 und $30 \mathrm{GE} / \mathrm{m}^{3}$ ) weichen die Merkmale deutlich voneinander $a b$.

Nach Abbildung 3 können $\mathrm{H}_{2} \mathrm{~S}$ und DMS durch Auswertung nur des Merkmals bei $230^{\circ} \mathrm{C}$ und mithilfe einer linearen Anpassung quantifiziert werden (Abbildung 4). Die Verwendung ausschließlich eines Merkmals beziehungsweise einer Temperaturstufe zur gemeinsamen Quantifizierung beider Gase ist jedoch wahrscheinlich nur unter Laborbedingungen möglich. Variationen der Hintergrundkonzentrationen haben möglicherweise einen Einfluss auf die gleiche Konzentrationsabhängigkeit der Merkmale für beide Probengase bei $230^{\circ} \mathrm{C}$ In der Praxis wird daher eine Temperaturstufe nicht ausreichen und wie in diesem Beitrag ein

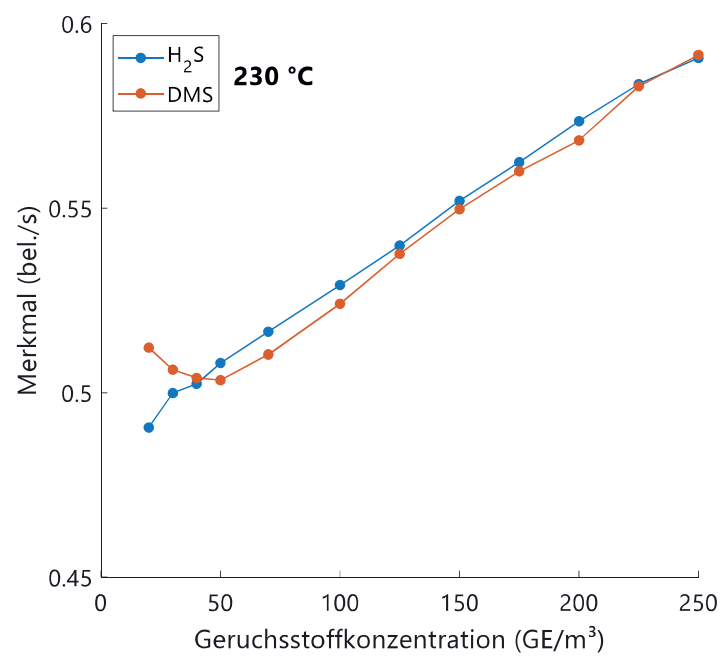

Abb.3: Konzentrationsabhängigkeit der DSRSteigung (gemittelt über mehrere Temperaturzyklen) beider Gase bei $230^{\circ} \mathrm{C}$. 


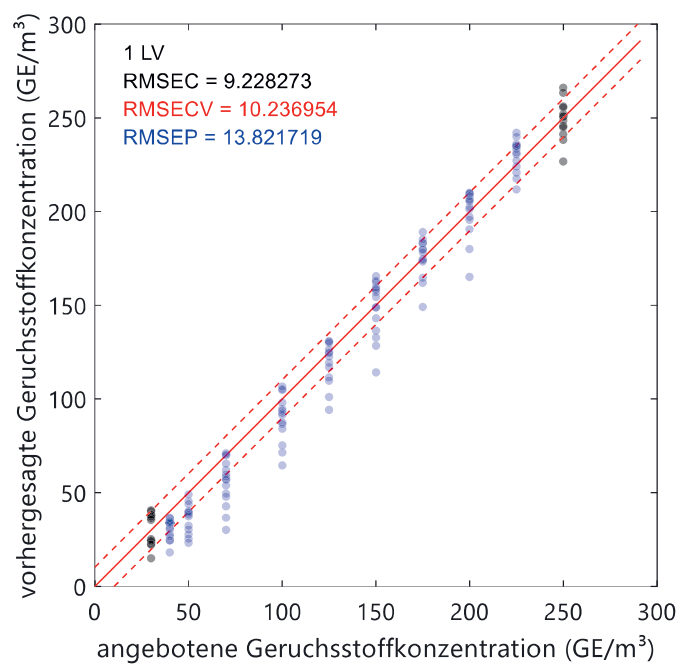

Abb. 4: $\quad$ Modell für $\mathrm{H}_{2} \mathrm{~S}$ und DMS gemeinsam über eine lineare Anpassung des Merkmals bei $230^{\circ} \mathrm{C}$. Die mittleren Konzentrationen (blaue Datenpunkte) wurden hierbei nach Training des Modells mit den äußersten Konzentrationen (schwarze Datenpunkte) vorhergesagt. Es zeigt sich eine gute Interpolierbarkeit.

Temperaturzyklus verwendet werden müssen, der eine selektive Messung ermöglicht und für die Quantifizierung mehr Merkmale zur Verfügung stellt. Die Quantifizierung kann dann mittels partial least squares regression (PLSR) erfolgen. Abbildung 5 zeigt die entsprechende Regression eines Einzelgases (Beispiel: $\mathrm{H}_{2} \mathrm{~S}$ in
Abbildung 5 a) und die Regression beider Gase gemeinsam (Abbildung 5 b). Die Fehler (RMSE, root mean square error) des Modelltrainings (RMSEC), der zehnfachen Kreuzvalidierung (RMSECV) und der Vorhersage nicht beim Training oder der Validierung verwendeter Datenpunkte (RMSEP) sind in den Abbildungen enthalten. Für die Einzelgase beträgt der RMSECV jeweils etwa $2 \mathrm{GE} / \mathrm{m}^{3}$ und der RMSEP jeweils circa $10 \mathrm{GE} / \mathrm{m}^{3}$. Für die gemeinsame Quantifizierung beider Gase liegen beide Werte bei etwa $11 \mathrm{GE} / \mathrm{m}^{3}$. Die Vorhersage der PLSR ist somit noch etwas besser als die der linearen Anpassung des Merkmals bei $230^{\circ} \mathrm{C}$ (RMSEP in etwa $14 \mathrm{GE} / \mathrm{m}^{3}$, Abbildung 4). Zudem ist zu erwarten, dass die Regression stabiler ist gegenüber Schwankungen des Hintergrundgases als ein einzelnes Merkmal.

Für die Quantifizierungen (Abbildungen 4 und 5) wurden nur die kleinste und die größte Konzentration zum Training verwendet, die Konzentrationen dazwischen wurden anschließend entsprechend des Modells vorhergesagt. Es zeigt sich hierbei eine sehr gute Interpolierbarkeit, was auch der kleine RMSEP verdeutlicht. Es können somit auch Konzentrationen gemessen werden, die nicht explizit kalibriert wurden.

Abbildung $5 \mathrm{~b}$ ist zusätzlich entsprechend eines gewählten Warnwertes von $100 \mathrm{GE} / \mathrm{m}^{3}$ farblich unterlegt. Grün kennzeichnet hierbei den Bereich unter $100 \mathrm{GE} / \mathrm{m}^{3}$, in dem eine Geruchsstoffkonzentration als ausreichend niedrig klassifiziert werden könnte. Oberhalb
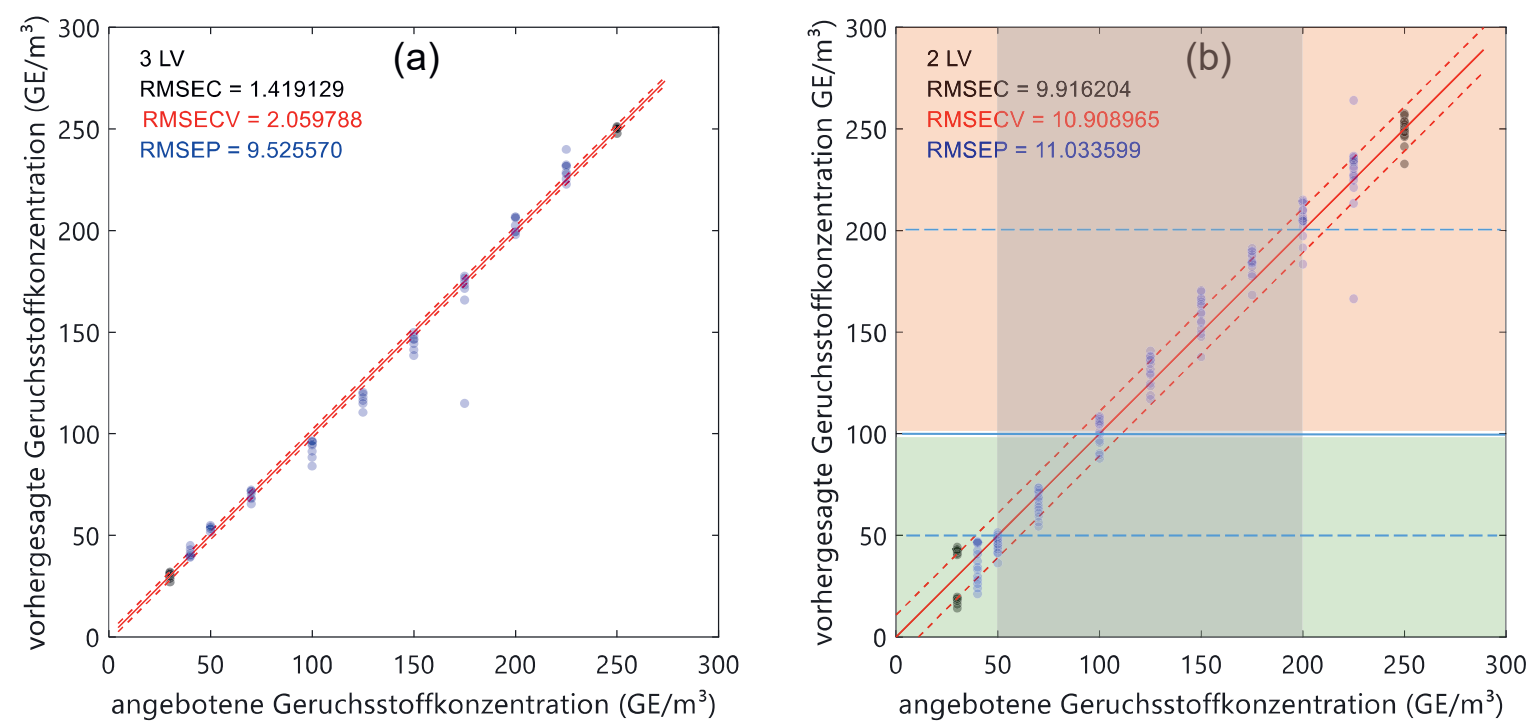

Abb. 5: $\quad$ PLSR für $\mathrm{H}_{2} \mathrm{~S}$ einzeln (a) sowie für $\mathrm{H}_{2} \mathrm{~S}$ und DMS gemeinsam (b). In beiden Fällen wurden alle Merkmale, $d$. $h$. alle Temperaturstufen, für die Regression verwendet. Zusätzlich ist in (b) der Bereich der erlaubten Unsicherheit bei der Bewertung bezüglich des Warnwertes von $100 \mathrm{GE} / \mathrm{m}^{3}$ grau unterlegt. Eine Fehlbewertung sehr kleiner (unter $50 \mathrm{GE} / \mathrm{m}^{3}$, grün) bzw. sehr großer Geruchsstoffkonzentrationen (über $200 \mathrm{GE} / \mathrm{m}^{3}$, rot) ist ausgeschlossen. 
des Warnwertes würde hingegen eine zu starke Geruchsemission erkannt werden (rot). Zwischen 50 und $200 \mathrm{GE} / \mathrm{m}^{3}$ (erlaubte Unsicherheit von Faktor zwei des Warnwertes, grauer Bereich) werden Fehlbewertungen erlaubt, außerhalb dieses Bereichs sollen falsche Vorhersagen jedoch ausgeschlossen werden. In der präsentierten Messung wird dies sicher erreicht.

Über die Merkmale sind die beiden Gase im untersuchten Konzentrationsbereich (20$250 \mathrm{GE} / \mathrm{m}^{3}$ ) außerdem mit einer linearen Diskriminanzanalyse (LDA) gut voneinander trennbar (Abbildung 6). Für Geruchsstoffkonzentrationen unter $50 \mathrm{GE} / \mathrm{m}^{3}$ liegen sie allerdings nahe beieinander, und $\mathrm{H}_{2} \mathrm{~S}$ ist für diese Konzentrationen nicht von Normallluft zu unterscheiden. Mit einem 5-Nächste-NachbarnKlassifikator und einer zehnfachen Kreuzvalidierung ergibt sich ein Klassifikationsfehler von etwa $12 \%$, zu dem vor allem die $\mathrm{H}_{2} \mathrm{~S}-$ Datenpunkte unter $50 \mathrm{GE} / \mathrm{m}^{3}$ beitragen, die der Normalluft zugeordnet werden.

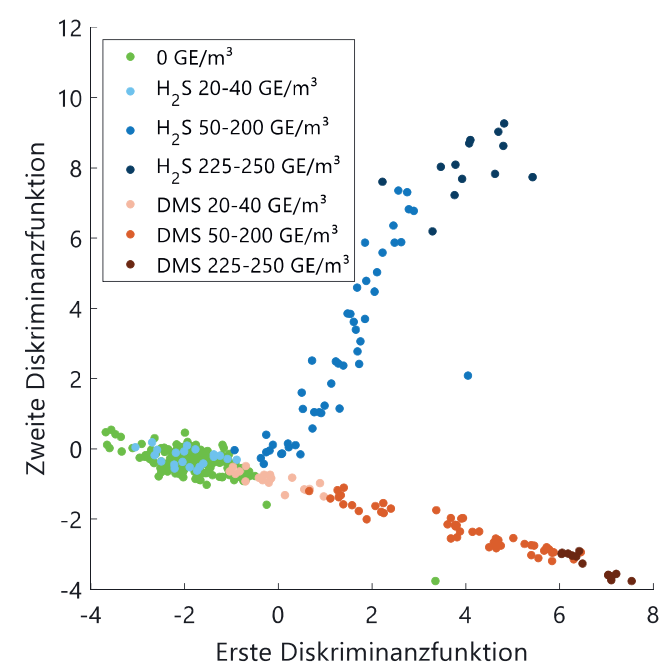

Abb. 6: Lineare Diskriminanzanalyse bezüglich der beiden Zielgase und des Hintergrunds.

\section{Zusammenfassung und Ausblick}

Es wurde gezeigt, dass durch geeignete Wahl des Temperaturzyklus sowohl die beiden Zielgase unterschieden werden können als auch eine gemeinsame Quantifizierung möglich ist. Außerdem reicht in diesem Fall schon ein Merkmal $\left(230^{\circ} \mathrm{C}\right)$ für eine gute Quantifizierung, mit einer Messunsicherheit kleiner $15 \mathrm{GE} / \mathrm{m}^{3}$. Das Überschreiten eines Schwellwertes von $100 \mathrm{GE} / \mathrm{m}^{3}$ mit einer erlaubten Unsicherheit von Faktor 2 kann somit sicher angezeigt werden. In der vorliegenden Messung wurde der Hintergrund nicht variiert. Ob der Hintergrund einen Einfluss auf die Übereinstimmung der
Empfindlichkeit für beide Gase bei $230{ }^{\circ} \mathrm{C}$ hat, muss noch untersucht werden.

Bei der präsentierten Untersuchung waren darüber hinaus die ähnlichen Geruchsschwellen der beiden Probengase von Vorteil. Eine Erweiterung des Verfahrens auf andere Gase, wie Methanthiol oder Dimethyldisulfid, deren Geruchsschwellen eine Größenordnung kleiner oder größer sind als die von $\mathrm{H}_{2} \mathrm{~S}$ und DMS, ist Gegenstand aktueller Untersuchungen.

\section{Danksagung}

Die Arbeiten wurden mit Mitteln des Bundesministeriums für Bildung und Forschung im Rahmen des Verbundprojekts SEPEG unter dem Förderkennzeichen 01 IS17087 gefördert. Wir danken den Projektpartnern 3S - Sensors, Signal Processing, Systems $\mathrm{GmbH}$, Saarbrücken, sowie Olfasense $\mathrm{GmbH}$, Kiel, für die wichtigen Diskussionsbeiträge und die gute Zusammenarbeit.

\section{Literaturnachweis}

[1] DIN EN 13725:2003-07, Luftbeschaffenheit Bestimmung der Geruchsstoffkonzentration mit dynamischer Olfaktometrie; Deutsche Fassung EN 13725:2003; doi: 10.31030/9364305

[2] DIN EN 16841-1:2017-03, Außenluft Bestimmung von Geruchsstoffimmissionen durch Begehungen - Teil 1: Rastermessung; Deutsche Fassung EN 16841-1:2016; doi: $10.31030 / 2503315$

[3] DIN EN 16841-2:2017-03, Außenluft Bestimmung von Geruchsstoffimmissionen durch Begehungen - Teil 2: Fahnenmessung; Deutsche Fassung EN 16841-2:2016; doi: 10.31030/2503331

[4] E. C. Sivret, G. Parcsi, R. M. Stuetz: Sampling and Analysis Methodology Concerns for Volatile Organo-Sulfur Compounds (VOSCs), Air and Waste Management Association Annual Conference and Exhibition 103, 5131 (2010)

[5] T. Baur, A. Schütze, T. Sauerwald: Optimierung des temperaturzyklischen Betriebs von Halbleitergassensoren, Tech. Mess. 82(4), 187195 (2015); doi: 10.1515/teme-2014-0007

[6] M. Leidinger, C. Schultealbert, J. Neu, A. Schütze, T. Sauerwald: Characterization and calibration of gas sensor systems at ppb level a versatile test gas generation system, Meas. Sci. Technol. 29(1), 015901 (2018); doi: 10.1088/1361-6501/aa91da

[7] T. Baur, C. Schultealbert, A. Schütze, T. Sauerwald: Device for the detection of short trace gas pulses, Tech. Mess. 85(7-8), 496-503 (2018); doi: 10.1515/teme-2017-0137

[8] T. Sauerwald, T. Baur, M. Leidinger, W. Reimringer, L. Spinelle, M. Gerboles, G. Kok, A. Schütze: Highly sensitive benzene detection with metal oxide semiconductor gas sensorsan inter-laboratory comparison, J. Sens. Sens. Syst. 7, 235-243 (2018); doi: 10.5194/jsss-7235-2018 
[9] M. Bastuck, T. Baur, A. Schütze: DAV3E - a MATLAB toolbox for multivariate sensor data evaluation, J. Sens. Sens. Syst. 7, 489-506 (2018); doi: 10.5194/jsss-7-489-2018
[10] Y. Nagata: Measurement of odor threshold by triangle odor bag method, Odor Meas. Rev. 118-127 (2003)

[11] Persönliche Mitteilung, Olfasense $\mathrm{GmbH}$ : Interne Datenbank zu Geruchsschwellen 